\title{
ANALYSIS OF BUNDLE ADJUSTMENTS AND EPIPOLAR MODEL ACCURACY ACCORDING TO FLIGHT PATH CHARACTERISTICS OF UAV
}

\author{
J. Son, P. Lim, J. Seo , T. Kim* \\ Dept. of Geoinformatic Engineering, Inha University, 100 Inharo, Namgu, Incheon, Republic of Korea \\ - json8520@inha.edu, vudco88@inha.edu,12141104@inha.edu, tezid@inha.ac.kr
}

KEY WORDS: UAV, Path characteristics, Bundle adjustment, Epipolar model, Exterior orientation

\begin{abstract}
:
Nowadays, UAVs(Unmanned aerial vehicles) are being used in spatial information construction. To construct spatial information, it is important to select appropriate data. Therefore, in this paper, we analyzed the characteristics of UAV flight paths and their effects on accuracy of bundle adjustments and epipolar models. We analyzed the flight path characteristics in three aspects: body stability, linearity of path and overlap between images. Firstly, in case of the body stability, ideal body stability is defined when images are taken in vertical direction. We calculated the body stability by the photographed angle, which is a difference between the vertical direction and actual photographed direction. Secondly, in case of the linearity of path, we are calculated the residual after fitting ground coordinates of orthogonal UAV positions or image centers by a straight line. Finally, in case of the overlap between images, we selected two closest images along vertical and horizontal direction as a pair and calculated the overlap of the pair on the ground space. We performed bundle adjustments and extracted EOPs(Exterior Orientation Parameters). Using the EOPs, we constructed an orthoimage, a DSM(Digital Surface model), and an epipolar model. By verified orthoimage and DSM, we were able to obtain accuracy of bundle adjustments. We also analyzed y-parallax of epipolar models to obtain its accuracy. Results showed that the body stability show very little affect vertical error of bundle adjustments. In case of linearity of path, there was little effect on the accuracy of bundle adjustment and epipolar models. The overlap between images affected all accuracy of bundle adjustments and epipolar model. The best accuracy was obtained when the overlap was about $70 \%$.
\end{abstract}

\section{INTRODUCTION}

Since a UAV(Unmanned aerial vehicle) has many advantages that other platforms cannot obtain, it has been used in many fields of military, communication, agriculture, transportation and so on. Particularly, a UAV can acquire high spatial and temporal resolution image data. From these advantages, its usage in a field of spatial information construction is increasing.

Lately, many studies about spatial information construction applying UAV was performed. Mancini (2013) has shown that it is possible to generate accurate high-resolution DSM (Digital Surface Model) using a UAV. Kim (2017) proposed image mosaicking method considering imaging characteristics of small UAV. Tsai and Lin (2017) has shown accelerated image matching technique for UAV orthoimage registration.

Although there are many studies about UAV image processing, studies on image quality analysis are still required. To construct spatial information stably and accurately, it is important to select appropriate data. Since a UAV has poor flight stability compared to other platforms, the flight path characteristics have a great influence on the UAV data quality. Therefore, in this paper, we analyzed the flight path characteristics and theirs effect on accuracy of bundle adjustments and epipolar models.

\section{PROPOSED METHOD}

\subsection{Flight Path Characteristics Analysis}

To analyze UAV data quality by flight path characteristics, we proposed three factors: body stability, linearity of path and overlap between images. Each flight path characteristic factor was analyzed based on collinearity condition models.

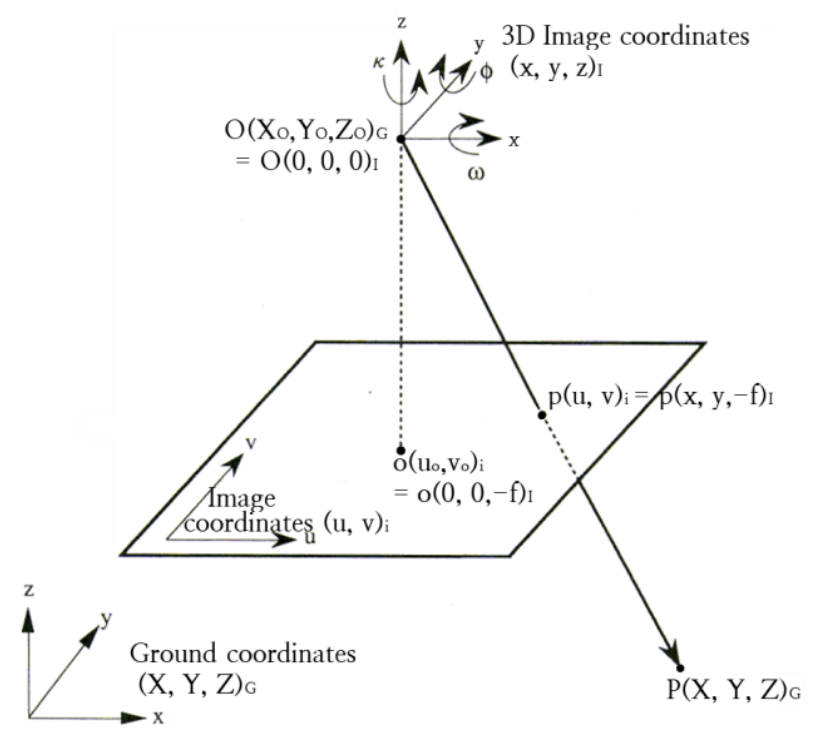

Figure 1. Colinear condition

\footnotetext{
* Corresponding author
} 
Collinearity condition is a physical model that describe geometric relationship between ground space and image space (Yuan, 1989). As shown in Figure 1, a line op connects perspective center and image point on image space and a line OP connects perspective center and ground point on ground space. two lines op and $\mathbf{O P}$ is on same line. Therefore, the following equation is established.

$$
\begin{aligned}
\text { OP } & =\lambda \cdot R_{I}^{G} \cdot \text { op } \\
\left(\begin{array}{c}
X-X_{O} \\
Y-Y_{O} \\
Z-Z_{O}
\end{array}\right) & =\lambda \cdot R_{I}^{G} \cdot\left(\begin{array}{c}
x \\
y \\
-f
\end{array}\right)
\end{aligned}
$$

where, $\lambda$ is scale

$\boldsymbol{R}_{I}^{G}$ is Rotation matrix

from Image space to ground space

from this equation, we were able to calculate a Ground point $(X, Y, Z)$ corresponding to image point $(x, y,-f)$. For this model, we needed IOP(Interior Orientation Parameter) and EOP(Exterior Orientation Parameter) that include position (Xo,Yo,Zo), and rotation $(\omega, \varphi, \kappa)$ of UAV.

2.1.1 Body Stability: Unlike other spatial information construction platforms such as satellite and aircraft, the body stability of UAV was relatively poor because of the weather conditions and flight characteristics. UAV is much sensitive to wind, rain, radio interference and others. Furthermore, some kinds of fixed-wing UAV have rolling rotation when flying.

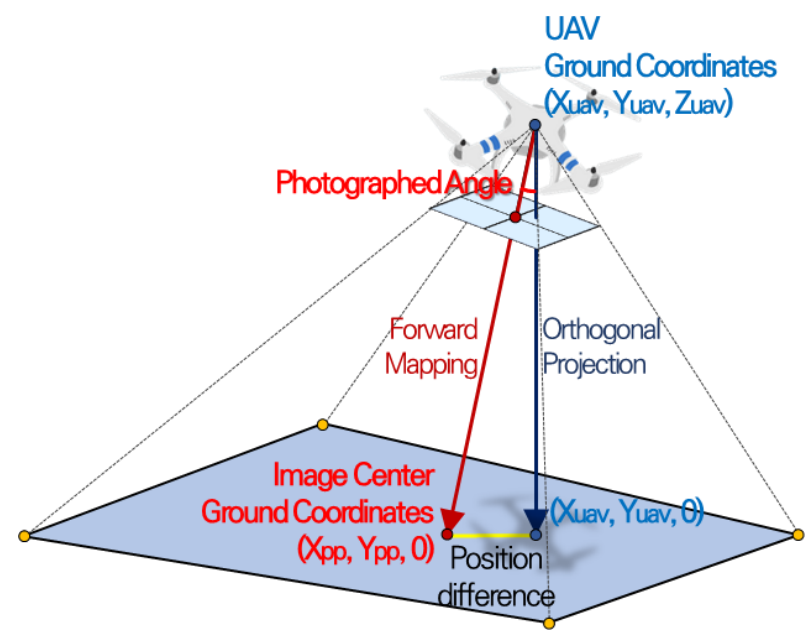

Figure 2. Body stability analysis

When the body stability is ideal, images are taken in vertical direction. However, it is impossible to take a vertical photograph image all the time. So, we calculated the body stability by the photographed angle, which is a difference between the vertical direction and the actual photographed direction.

To calculate this photographed angel, we conducted following process. Firstly, we conducted orthogonal projection of UAV`s position available in EOPs. Secondly, we constructed the collinear condition modeling and calculated ground coordinates of image center. Finally, we calculated the photographed angle through the ratio between position difference of the two coordinates and flight altitude.

$$
\text { Photographed Angle }=\operatorname{atan}\left(\frac{\text { Position difference }}{\text { Flight Altitude }}\right)
$$

2.1.2 Linearity of Path: Most UAVs acquire images according to a pre-planned linear path. Therefore, linearity of path can be an indicator of how well the pre-planned imaging plan was performed.

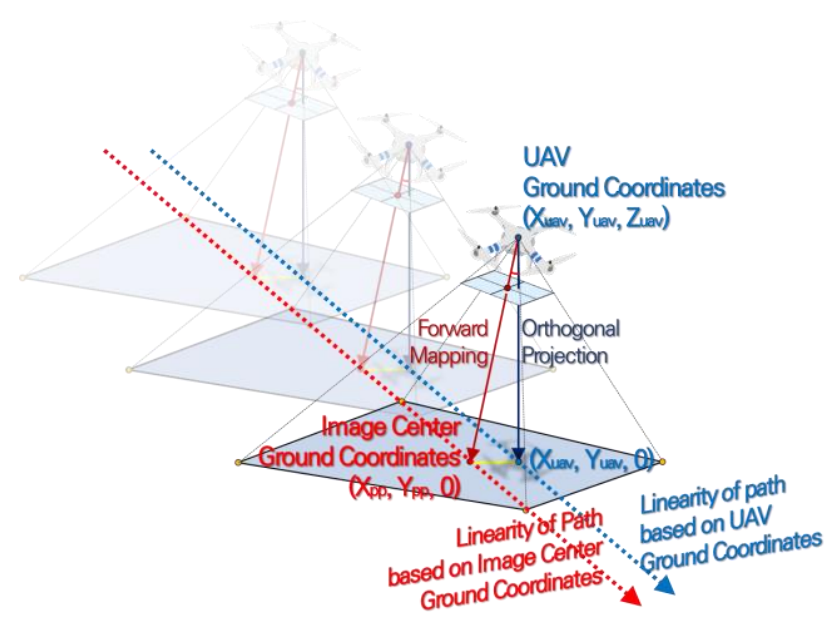

Figure 3. Linearity of path analysis

In this paper, we analyzed linearity based on two coordinates: image center ground coordinates and orthogonal UAV ground coordinates. Analysis based on the orthogonal UAV ground coordinates shows the linearity of the UAV flight path and analysis based on the image center ground coordinates shows the linearity of the photographed image.

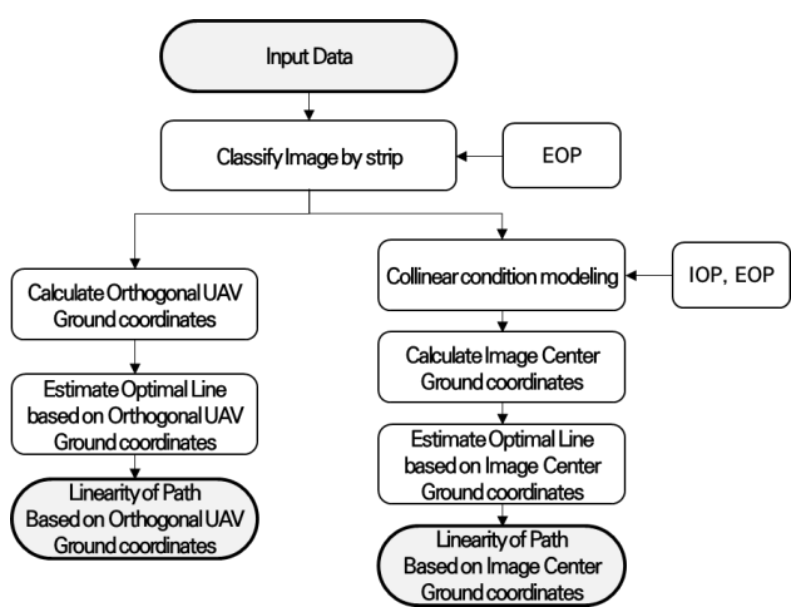

Figure 4. Linearity of path analysis process

The linearity analyze process consists of three steps. First steps, we classified the images by strip using the horizontal position and kappa angle of the EOP. We then removed images photographed during rotation between strips. Second steps, Using IOP and EOP of each image, we performed the collinearity condition modeling and then we calculated ground coordinates of the image center. We also calculated orthogonal projected ground coordinates of UAV. Final step, we are calculated the residual after fitting the calculated ground coordinates of orthogonal UAV position or image center by a 
straight line. Through the average of the residuals, we determined the linearity of path.

2.1.3 Overlap: Many previous studies have shown effect of overlap between images in spatial information construction using satellite or aircraft image data (Zebedin et al., 2006; Botterill et al., 2010). But there are few studies to analyze the overlap in UAV images for spatial information construction.

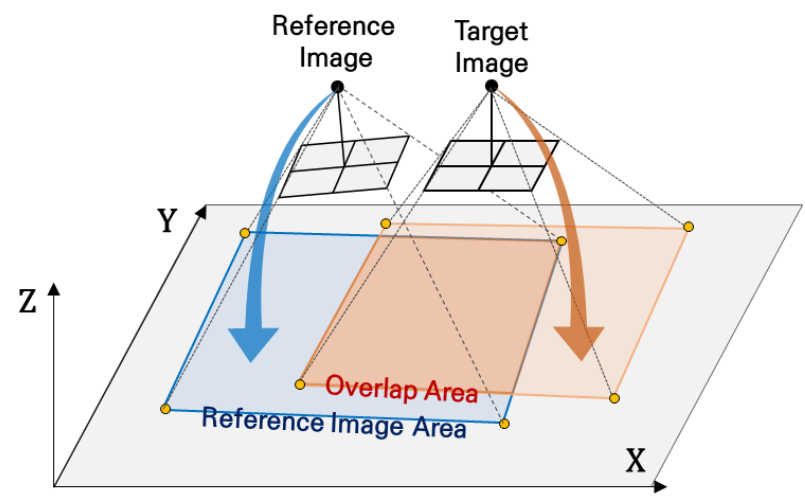

Figure 5. Overlap between images analysis

As shown in Figure 5, in an image pair, we defined one image as a reference image and calculated the overlap by a ratio of an overlapped area over the reference image area in ground space.

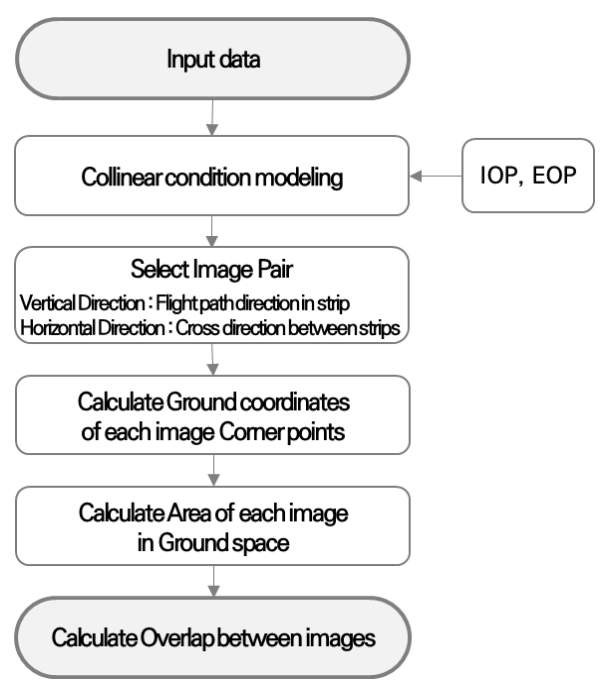

Figure 6. Overlap between images analysis process

We constructed the overlap calculation process as follow. Firstly, we performed modelling the relationship between ground coordinate system and image coordinate system based on the collinearity condition. Secondly, we selected image pairs. In the same strip, according to the photograph sequence, the front and back images were selected as one pair. Or we selected two closest images on adjacent strips as one pair. Thirdly, we calculated ground coordinates of each image corner points. Fourthly, we calculated area of each image in ground space. Finally based on each calculated area, we determined overlap between images.

\subsection{Bundle Adjustments and Epipolar Model}

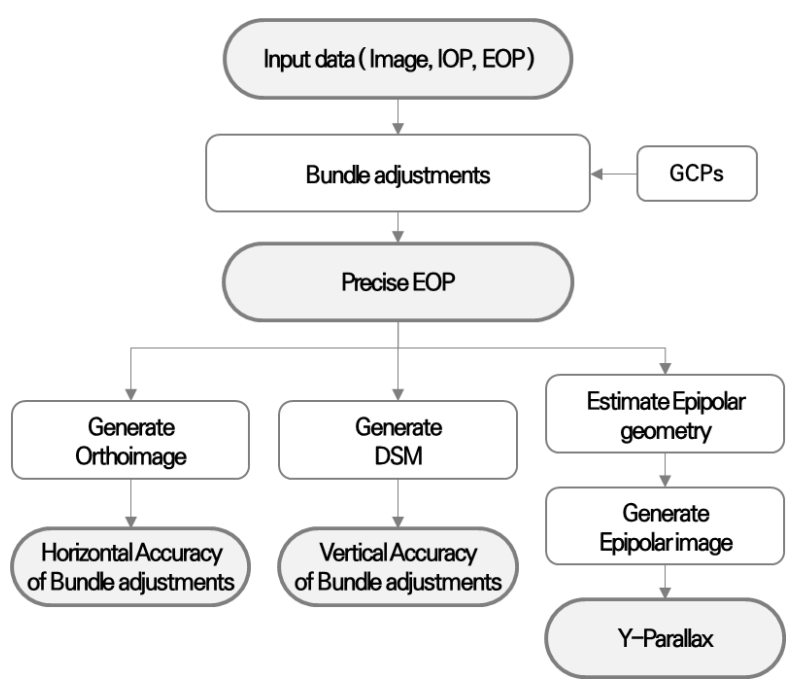

Figure 7. Overlap between images analysis process

Bundle adjustments is a method precisely adjust orientation of image based on collinear condition. Most of spatial information construction using image is based on the bundle adjustments. In order to analyze the relationship between the flight path characteristic and accuracy of bundle adjustments, we performed bundle adjustment and construct orthoimage and DSM(Digital Surface Model) using a UAV image processing software. And then using validation points, we calculated vertical accuracy in DSM and horizontal accuracy in orthoimage.

Precision digital mapping is mostly performed through stereo plotting. To improve the accuracy of stereo plotting, the epipolar model accuracy is important. Epipolar modeling is the procedure of eliminating vertical disparity between stereo images(Kim and Kim, 2016). As an ideal epipolar model has yparallax of 0 , we can determine epipolar model accuracy with yparallax. As a result of bundle adjustment, we were able to obtain precise EOPs. Using these EOPs, we estimated epipolar model and created epipolar images. By analyzing the Y parallax of the epipolar image, epipolar model accuracy was calculated.

\section{EXPELIMENTS AND ANALYSIS}

\subsection{Experiment Data}

\begin{tabular}{l|ccc}
\hline \hline UAV Model & eBee & Inspire2 & FireFly6 \\
\hline UAV Type & fixed-wing & rotary-wing & hybrid \\
\hline Camera & $\begin{array}{c}\text { S.O.D.A } \\
\text { Camera }\end{array}$ & $\begin{array}{c}\text { Zenmuse } \\
\text { X5S }\end{array}$ & $\begin{array}{c}\text { SONY } \\
\text { a6000 }\end{array}$ \\
\hline Number of Datasets & 4 & 4 & 3 \\
\hline photographed area & $0.5 \mathrm{~km}^{2}$ & $0.5 \mathrm{~km}^{2}$ & $0.5 \mathrm{~km}^{2}$ \\
\hline GSD & $5 \mathrm{~cm}$ & $5 \mathrm{~cm}$ & $5 \mathrm{~cm}$ \\
\hline \hline
\end{tabular}

Table 1. Experiment data

For experiments, we used three UAVs. One was eBee, which is a fixed-wing UAV. Another was Inspire2, which is a rotarywing UAV, and the other was FireFly 6, which is a hybrid UAV. 


\begin{tabular}{|c|c|c|c|c|c|c|c|c|}
\hline \multirow[b]{2}{*}{ dataset } & \multirow[b]{2}{*}{ UAV } & \multirow{2}{*}{$\begin{array}{c}\text { Body } \\
\text { Stability } \\
\begin{array}{c}\text { Photographed } \\
\text { Angle } \\
\text { (Deg.) }\end{array}\end{array}$} & \multicolumn{2}{|c|}{$\begin{array}{l}\text { Linearity of Path } \\
\text { (m) }\end{array}$} & \multirow{2}{*}{$\begin{array}{c}\text { Overlap } \\
\text { between } \\
\text { images } \\
(\%)\end{array}$} & \multicolumn{2}{|c|}{$\begin{array}{c}\text { Bundle adjustment } \\
\text { Accuracy } \\
(\mathrm{m})\end{array}$} & \multirow{2}{*}{$\begin{array}{c}\text { Epipolar } \\
\text { Model } \\
\text { Accuracy }\end{array}$} \\
\hline & & & $\begin{array}{c}\text { Based on } \\
\text { Orthogonal } \\
\text { UAV } \\
\text { Coordinates } \\
\end{array}$ & $\begin{array}{c}\text { Base on } \\
\text { image center } \\
\text { Coordinates }\end{array}$ & & $\begin{array}{l}\text { Horizontal } \\
\text { Accuracy }\end{array}$ & $\begin{array}{l}\text { Vertical } \\
\text { Accuracy }\end{array}$ & \\
\hline 1 & \multirow{4}{*}{ eBee } & 11.45 & 1.58 & 16.49 & 50.55 & 0.045 & 0.127 & 5.8439 \\
\hline 2 & & 12.06 & 2.59 & 25.76 & 50.71 & 0.053 & 0.032 & 28.1987 \\
\hline 3 & & 12.23 & 2.31 & 25.36 & 50.49 & 0.028 & 0.032 & 5.8439 \\
\hline 4 & & 9.81 & 0.9 & 11.8 & 52.085 & 0.054 & 0.054 & 21.112 \\
\hline 5 & \multirow{4}{*}{ Inspire2 } & 3.7 & 0.58 & 3.35 & 75.395 & 0.048 & 0.029 & 4.6085 \\
\hline 6 & & 3.69 & 0.79 & 2.77 & 76.385 & 0.041 & 0.025 & 6.8639 \\
\hline 7 & & 1.61 & 0.59 & 0.76 & 80.74 & 0.069 & 0.034 & 13.2448 \\
\hline 8 & & 1.69 & 0.87 & 2.05 & 79.71 & 0.054 & 0.034 & 24.8454 \\
\hline 9 & \multirow{3}{*}{ FireFly6 } & 3.9 & 1.88 & 6.55 & 62.755 & 0.036 & 0.022 & 9.384 \\
\hline 10 & & 4.51 & 3.67 & 17.11 & 59.57 & 0.041 & 0.017 & 11.7285 \\
\hline 11 & & 4.92 & 4.82 & 21.87 & 61.915 & 0.038 & 0.019 & 9.0674 \\
\hline
\end{tabular}

Table 2. Experiment results

We used 11 UAV datasets: 4 datasets acquired from eBee, 4 datasets acquired from Inspire2, 3 datasets acquired from FireFly6. All datasets have $0.5 \mathrm{~km}^{2}$ photographed area and $5 \mathrm{~cm}$ GSD.

To perform bundle adjustment, we used 5 GCPs in each data. And to analyze the accuracy of bundle adjustment, 3 validation points for each dataset was used.

\subsection{Experiment Results}

Above Table 2 show the calculated three flight path characteristics and accuracy of bundle adjustments and epipolar model of used 11 UAV datasets. In case of body stability, the Inspire2 showed good performance. Since it has gimbal, orientation stability of sensor was much higher than others. On the other hand, eBee showed the poor body stability. This was due to the nature of fixed-wing UAV flight characteristic.

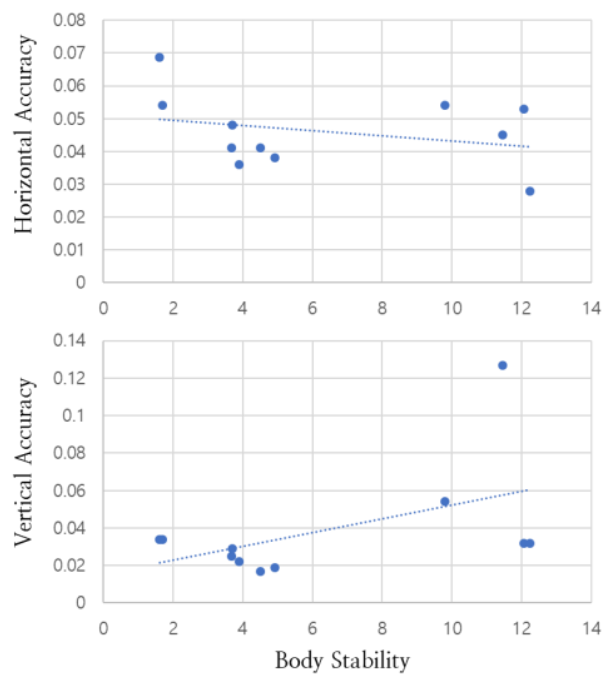

Figure 8 . Relationship between body stability and accuracy of bundle adjustment
Figure 8 show the relationship between body stability and accuracy of bundle adjustment. In this result, we could not find obvious relationship between body stability and accuracy of bundle adjustments. very few relationships between body stability and vertical accuracy was found.

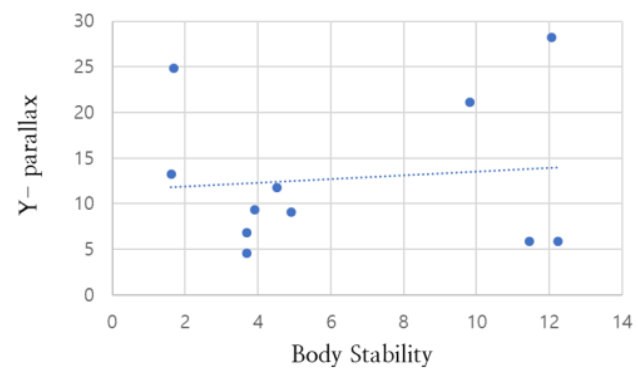

Figure 9. Relationship between body stability and accuracy of epipolar model

As shown in Figure 9, there was no distinct relationship between body stability and accuracy of epipolar model. Therefore, body stability did not affect accuracy of bundle adjustments and epipolar models.

In case of linearity of path, Inspire 2 datasets show the best linearity based on orthogonal UAV coordinates and images center coordinates. eBee is showed small linearity error when we analyzed linearity based on orthogonal UAV coordinates, but huge errors when we analyzed linearity based on image center coordinates. This is due to the nature of a fixed-wing UAV. 

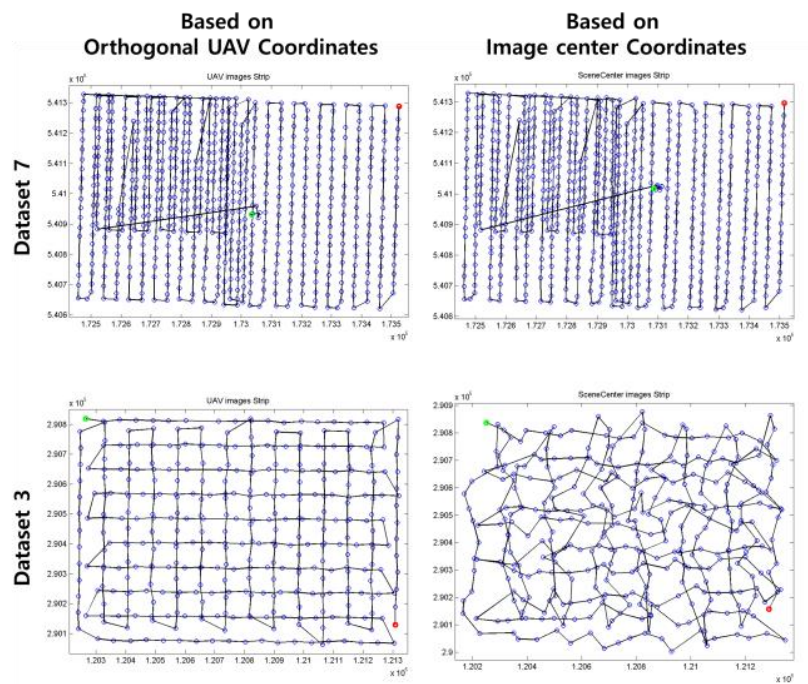

Figure 10. Path linearity of dataset 3(from eBee) and 7(from Inspire2)

As shown in Figure 10, dataset 7 show the better linearity based on both orthogonal UAV coordinates and image center coordinates. However, dataset 7 show worse performance than dataset 3 in all accuracy of bundle adjustment and epipolar model. Furthermore, we analyzed scatterplot between linearity of path and accuracy of bundle adjustment and epipolar model (Figure 11). We could not find distinct relationship. Therefore, the linearity of path does not have effect on bundle adjustments and epipolar modeling.
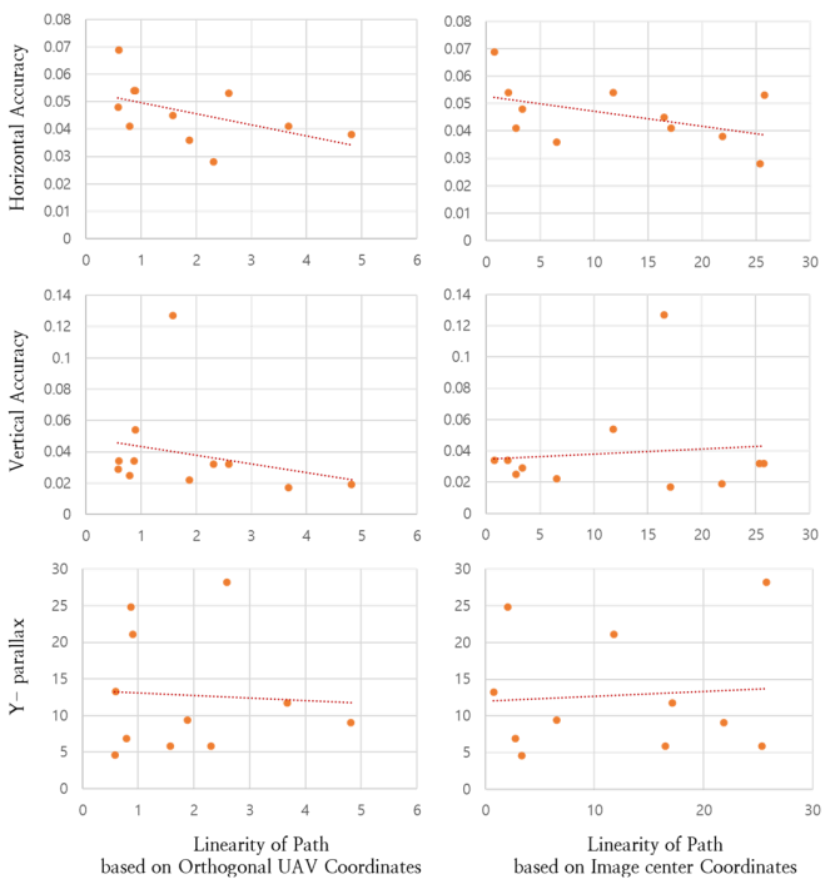

Figure 11. Relationship between linearity of path and accuracy of bundle adjustment and epipolar model

In case of overlap between images, as shown in Figure 12, we were able to find the trend that all accuracy of bundle adjustments and epipolar model was improved until overlap was $70 \%$. It was found that the accuracy of bundle adjustments and epipolar model is rather reduced when the overlap increases more than $70 \%$.
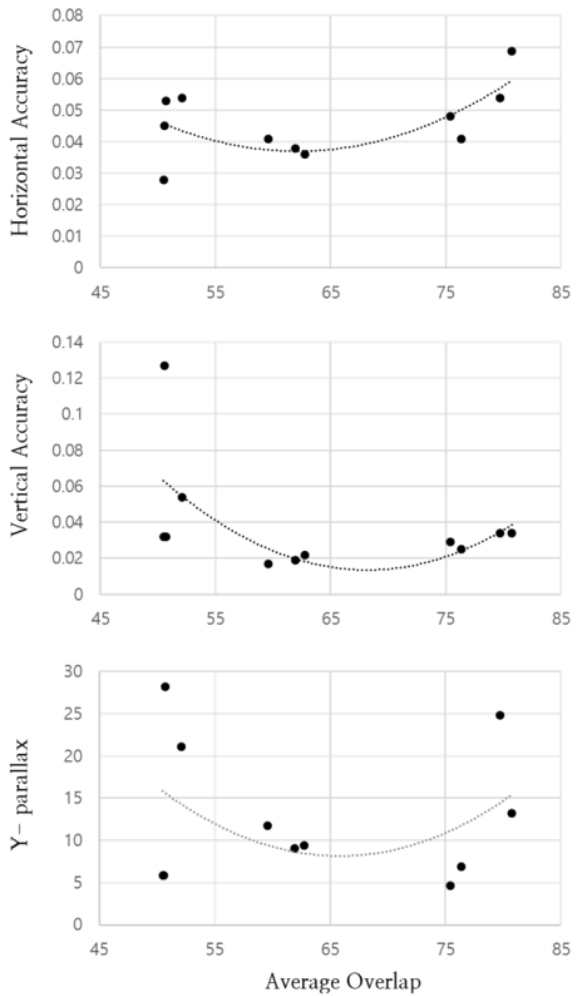

Figure 12. Relationship between average Overlap and accuracy of bundle adjustment and epipolar model

In the same photographed altitude, to increase the overlap between images, baseline should be decreased. As baseline decreased, the convergence angle is decreased at the same time. Previous studies have shown that bundle adjustments performance is poor in stereo image pair with too small convergence angle (Jeong et al., 2016).

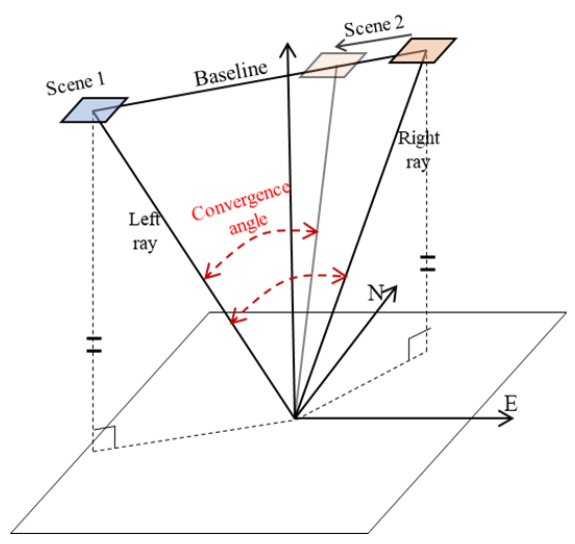

Figure 13. Example of convergence angle

\section{CONCLUSION}

In this paper, we analyzed flight path characteristics of UAV data with three factors and its effect on bundle adjustments and epipolar model. As a result, fixed-wing UAV showed relatively poor body stability because of it flight characteristics. the body stability did not affected accuracy of bundle adjustments and 
epipolar resampling. In case of linearity of path, there is no distinct relationship between Linearity and accuracy of bundle adjustments and epipolar model. The overlap between images affects all accuracy of bundle adjustments and epipolar model. The accuracy was increased until overlap was $70 \%$. If the overlap exceeded $70 \%$, the accuracy was rather reduced. It is because the convergence angle became too small.

Since almost UAV image coverage is small unlike satellite and aircraft image, convergence angle is more sensitive to overlap. Therefore, in future research, we will analyze the effect of convergence angle in UAV data and develop the method to define the best photographed condition included overlap, body stability, and convergence angle.

\section{ACKNOWLEDGEMENTS}

This study was carried out with the support of "Cooperative Research Program for Agriculture Science \& Technology Development (PJ01350003)" Rural Development Administration, Republic of Korea and with the support of "National Geographic Information Institute", Republic of Korea

\section{REFERENCES}

Botterill, T., Mills, S., Green, R., 2010. Real-time aerial image mosaicing. In 2010 25th International Conference of Image and Vision Computing, New Zealand, pp. 1-8.

Jeong, J., Kim, T., 2016. Quantitative estimation and validation of the effects of the convergence, bisector elevation, and asymmetry angles on the positioning accuracies of satellite stereo pairs. Photogrammetric Engineering \& Remote Sensing, Vol.82, Issue 8, pp. 625-633.

Kim, J. I., Kim, T., 2016. Comparison of computer vision and photogrammetric approaches for epipolar resampling of image sequence. Sensors, Vol.16, no. 3, pp. 412.

Kim, J., 2017. Development of a Tiepoint Based Image Mosaicking Method Considering Imaging Characteristics of Small UAV, Ph.D. dissertation, Inha University, Incheon, Korea, pp.1-27.

Mancini, F., Dubbini, M., Gattelli, M., Stecchi, F., Fabbri, S., Gabbianelli, G., 2013. Using unmanned aerial vehicles (UAV) for high-resolution reconstruction of topography: The structure from motion approach on coastal environments. Remote Sensing, Vol 12, pp. 6880-6898.

Tsai, C.H., Lin, Y.C., 2017. An accelerated image matching technique for UAV orthoimage registration. ISPRS Journal of Photogrammetry and Remote Sensing, Vol.128, pp. 130-145.

Yuan, J.C., 1989. A general photogrammetric method for determining object position and orientation. ieee Transactions on Robotics and Automation, Vol.5, Issue 2, pp.129-142.

Zebedin, L., Klaus, A., Gruber-Geymayer, B., Karner, K., 2006. Towards 3D map generation from digital aerial images. ISPRS Journal of Photogrammetry and Remote Sensing, Vol.60, Issue 6, pp. 413-427. 